\title{
Retinal angiographic alteration in diabetic macular edema after dexamethasone implantation: a case
}

\section{report}

This article was published in the following Dove Press journal:

International Medical Case Reports Journal

\author{
Chia-Yi Lee ${ }^{1,2, *}$ \\ Cheng-Chao Ching ${ }^{1} *$ \\ Chi-Chin Sun ${ }^{3,4}$ \\ Hung-Chi Chen ${ }^{5-7}$ \\ Hung-Yu Lin ${ }^{1,8-11}$
}

'Department of Ophthalmology, Show Chwan Memorial Hospital, Changhua, Taiwan;

${ }^{2}$ Department of Optometry, College of Medicine and Life Science, Chung Hwa

University of Medical Technology, Tainan, Taiwan; ${ }^{3}$ Department of Ophthalmology, Chang

Gung Memorial Hospital, Keelung, Taiwan;

${ }^{4}$ Department of Chinese Medicine, Chang

Gung University, Taoyuan City, Taiwan;

${ }^{5}$ Department of Ophthalmology, Chang Gung

Memorial Hospital, Linkou, Taiwan;

${ }^{6}$ Department of Medicine, Chang Gung

University College of Medicine, Taoyuan,

Taiwan; ${ }^{7}$ Center for Tissue Engineering, Chang

Gung Memorial Hospital, Linkou, Taiwan;

${ }^{8}$ Institute of Medicine, Chung Shan Medical

University, Taichung, Taiwan; ${ }^{9}$ Department of

Optometry, Chung Shan Medical University,

Taichung, Taiwan; ${ }^{10}$ Department of Optometry,

Yuanpei University of Medical Technology,

Hsinchu, Taiwan; " Department of Exercise and

Health Promotion, Chung Chou University of

Science and Technology, Changhua, Taiwan

*These authors contributed equally to this work

Correspondence: Hung-Chi Chen Department of Ophthalmology, Chang

Gung Memorial Hospital, 5 Fuxing Street,

Guishan District, Taoyuan 33305, Taiwan

Tel +886 3328 I200 ext. 8674

Fax +8863328 7798

Email mr3756@cgmh.org.tw

Hung-Yu Lin

Department of Ophthalmology, Show

Chwan Memorial Hospital, No.2, Lane

530, Section I, Zhongshan Road,

Changhua City, Changhua County 50093,

Taiwan

Tel +8864712 I452

Email anthonyhungyulin@hotmail.com

\begin{abstract}
Here we reported a rare case of the implantation of a dexamethasone intravitreal implant (DEX) in which decreased retinal vessel density (VD) was found by optical coherence tomography angiography (OCTA). A 74-year-old male with diabetes mellitus presented with bilateral macular edema. The best-corrected visual acuity (BCVA) was 0.6 in the right eye. Diabetic macular edema (DME) was diagnosed. A DEX for the right eye was planned, and the preoperative evaluation showed a superficial VD of 48.74 percent, a deep VD of 53.12 percent, and a foveal avascular zone (FAZ) $0.165 \mathrm{~mm}^{2}$ in size by OCTA. The BCVA in the right eye recovered to 0.8 , and a notably lower superficial VD of 45.97 percent and a deep VD of 45.40 percent were observed with an enlarged FAZ of $0.294 \mathrm{~mm}^{2}$ one month postoperatively. Moreover, BCVA in the right eye was maintained at 0.8 , while further reductions in both superficial (40.07 percent) and deep (40.91 percent) VD were noted with a FAZ measured at $0.305 \mathrm{~mm}^{2}$ two months postoperatively. In conclusion, retinal superficial and deep VD decreased, while the FAZ increased, after the implantation of the DEX in a patient with DME.
\end{abstract}

Keywords: dexamethasone, Ozurdex, optical coherence tomography angiography, diabetic macular edema, retina

\section{Introduction}

Diabetic macular edema (DME) will lead to the progressive reduction in central vision and poor quality of life. ${ }^{1}$ The dys-regulation of vessel growth and inflammatory cytokines are the major pathophysiological mechanisms of DME and lead to increased central macular thickness $(\mathrm{CMT}){ }^{2}$ Concerning the retinal image, both fluorescein angiography and optical coherence tomography angiography (OCTA) revealed the loss of a regular pattern in the retinal capillary plexus. ${ }^{3,4}$ The conventional treatments for DME, including intravitreal corticosteroid and intravitreal antiangiogenesis agent injections, have different outcomes. ${ }^{1}$

Dexamethasone intravitreal implant (DEX, brand name: Ozurdex) is a biodegradable steroid delivery system that has been widely applied to patients with DME with favorable morphological and functional outcomes. ${ }^{5}$ In clinical practice, the DEX was commonly implanted and with a pro re nata treatment approximately five months after the first implantation, and the long-term efficiency and safety was guaranteed in repeated DEX implantation. ${ }^{6}$ In a previous study, DEX reduces the CMT more effectively than anti- angiogenesis agents with significant improvement 
in retinal vascular caliber. ${ }^{7}$ Moreover, the DEX can be used to treat macular edema that shows a poor response to anti- angiogenesis agents with satisfactory results. ${ }^{8}$

Although the main theory of the use of DEX for treating DME is its anti-inflammatory effect, ${ }^{2}$ vascular endothelial growth factor is also suppressed by corticosteroids. ${ }^{2,5}$ Moreover, the different response of each retinal vascular layer to anti- angiogenesis therapy indicates that similar vascular alterations may also occur in patients receiving DEX implantation. ${ }^{9}$ Nevertheless, only one study demonstrated a change in the OCTA vasculature in patients who received DEX implantation with DME with insignificant alterations. ${ }^{10}$ In this study, we aimed to report a case of DME in which decreased retinal vessel density (VD) was found by OCTA after DEX implantation.

\section{Case report}

A 74-year-old Taiwanese male with diabetes mellitus presented with progressive blurred vision of both eyes. On examination, the best-corrected visual acuity (BCVA) was 0.6 in the right eye and 0.7 in the left eye with an intraocular pressure of $11 \mathrm{mmHg}$ in both eyes. Bilateral macular edema was observed using an indirect fundoscopy. Color fundus photography and fluorescein angiography revealed an edematous macula and vessel leakage; thus, DME was diagnosed (Figure 1). For the DME in the right eye, DEX injection was planned for the right eye, and the preoperative evaluation showed a CMT of $385 \mu \mathrm{M}$, and a superficial VD of 48.74 percent, a deep VD of 53.12 percent and a foveal avascular zone (FAZ) of $0.165 \mathrm{~mm}^{2}$ (Figure 2). The surgery was performed smoothly. One month after surgery, BCVA in the right eye recovered to 0.8 , and the CMT decreased to $292 \mu \mathrm{M}$. Moreover, a considerably lower superficial VD of 45.97 percent and a deep VD of 45.40 percent were observed with an enlarged FAZ of $0.294 \mathrm{~mm}^{2}$ (Figure 3 ).
In the latest follow-up visit two months postoperatively, BCVA in the right eye was maintained at 0.8 with a CMT of $281 \mu \mathrm{M}$. VD further declined in both superficial (40.07 percent) and deep (40.91 percent) areas, while the FAZ measured $0.305 \mathrm{~mm}^{2}$, which was larger than previously observed (Figure 4). In addition, the VD of the choriocapillaris in the right eye decreased from 2.103 to $1.853 \mathrm{~mm}^{2}$.

\section{Discussion}

In our patient, we used the same OCTA device (Angiovue, Optovue Inc., Bayview, CA, USA) in all OCTA examination, and the vessel layers was segmented manually by one ophthalmologist (HC Chen) then the VD and FAZ were calculated by the software in the same OCTA device. The results showed a decrement of VD and increment of the FAZ area after the DEX implantation. OCTA has been used to evaluate the retinal vessel for years, and its accuracy is similar to that of fluorescein angiography. ${ }^{3,11}$ Moreover, cystoid macular edema and DME were examined by OCTA in previous studies with a significant alteration of macular VD. ${ }^{12,13}$ The distribution of VD in the superficial and deep retinal layers was observed in a patient with DME who received intravitreal anti-angiogenesis agent injection via OCTA. ${ }^{14}$ As a result, it is reasonable to use OCTA to evaluate the changes in the macular vasculature in layers after receiving DEX intravitreal therapy in patients diagnosed with DME. Still, the segmentation artifacts would become more prominent in patients with macular edema and results in poorer interpretability of OCTA image, ${ }^{15}$ which can occur in the current study. Further effort to decrease such artifact in OCTA device is necessary.

DEX is a long-term steroid-release system that has a pen-like appearance that consists of the delivery device and dexamethasone medication (Figure 5). Upon injection, the

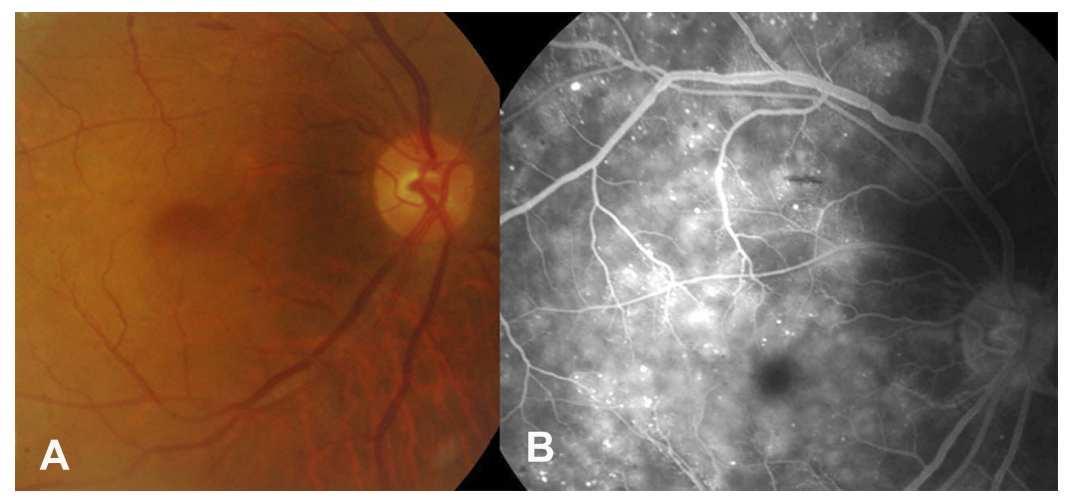

Figure I Photograph of the fundus in the case with diabetic macular edema. (A) Color fundus photography. (B) Fluorescein angiography. 


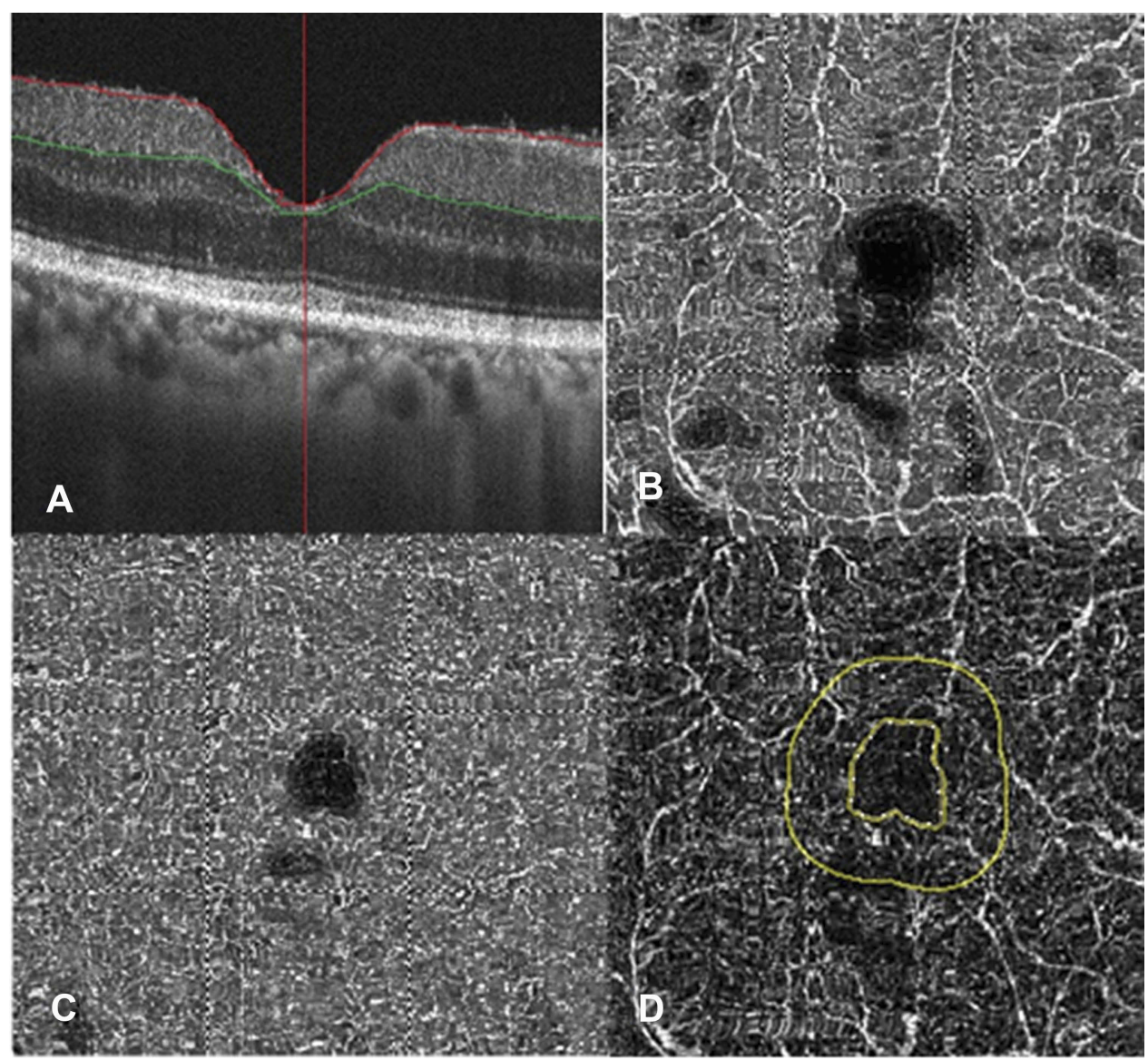

Figure 2 Macular conditions before dexamethasone implantation via optical coherence tomography angiography device. (A) Foveal thickness. (B) Superficial vessel density. (C) Deep vessel density. (D) Foveal avascular zone.

dexamethasone-contain polymer device (arrow, Figure 5) would be delivered into the vitreous cavity and release the dexamethasone continuously. The previous experimental study revealed that the tumor-induced angiogenesis diminished after dexamethasone application, ${ }^{16}$ implying the possibility of a steroid-induced anti-vascular growth effect in the eye. Only one study evaluated the change in the vasculature in patients with DME after DEX implantation without significant changes among the superficial VD, deep VD and choriocapillaris capillary density, and the difference was within two percent. ${ }^{10}$ In addition, another study also revealed non-significant alteration of vascular distribution in patients with retinal venous occlusions after the implantation of DEX. ${ }^{17}$ However, a decrease in the superficial and deep VDs of approximately 20 percent after DEX implantation was observed in the current study, while the enlargement of the FAZ found in the current study was not reported in previous studies. The prominent difference in vessel alteration and the FAZ indicated that the retinal vasculature may change in some patients with DME after DEX implantation. Although diabetic retinopathy would uneventfully enlarge the FAZ, which progressed with the disease severity, ${ }^{18}$ no severe complications, such as vitreous hemorrhage or tractional retinal detachment, were found during the two-month period. In addition, the blood sugar level was $212 \mathrm{mg} / \mathrm{dL}$ and $171 \mathrm{mg} / \mathrm{dL}$ before and two months after the DEX implantation, and the glycosylated hemoglobin was 7.3 percent and 7.0 percent before and two months after the DEX implantation, implied that the progression of diabetes mellitus was not likely. Moreover, the enlarged FAZ area in diabetic retinopathy and retinal venous occlusion are associated with worse visual outcome according to previous research, ${ }^{19}$ while the recovered visual acuity is discordant to the enlarged FAZ area in our patient, which further demonstrated that the enlarged FAZ would probably result from the anti-angiogenesis effect of DEX, rather than the influence of diabetic retinopathy. As a consequence, we speculated that the anti-angiogenesis effect of DEX, which proven in experimental study, ${ }^{16}$ retarded the angiogenesis process and lead to the decrement of retinal vasculature in 


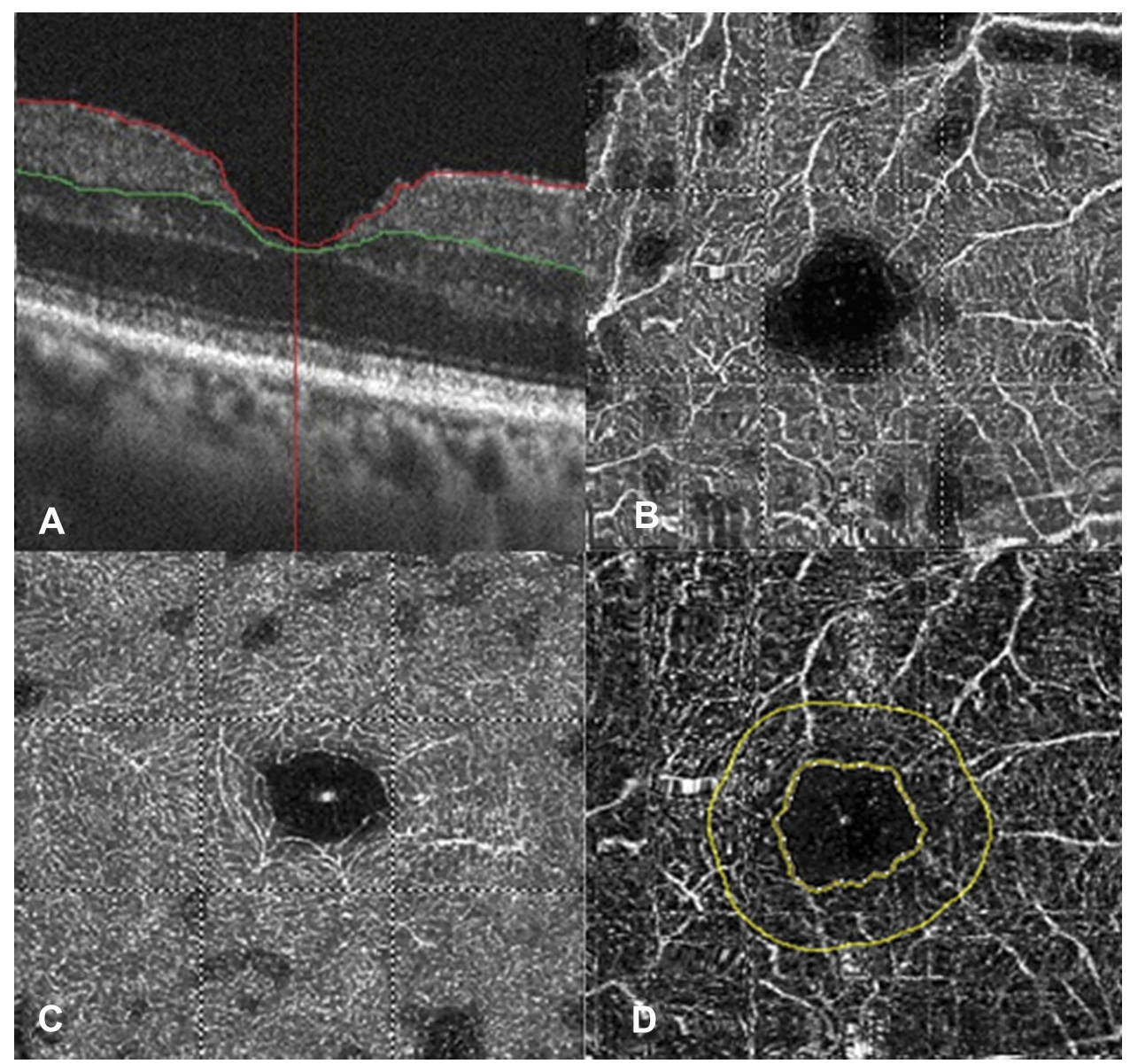

Figure 3 Macular conditions one month after dexamethasone implantation assessed an optical coherence tomography angiography device. (A) Foveal thickness. (B) Superficial vessel density. (C) Deep vessel density. (D) Foveal avascular zone.

our patient which presented as decreased VD and enlarged FAZ compared to the minimal change in the fellow eye. In addition, the effect of anti-angiogenesis persisted for months which may due to the long-term effect of DEX. Conversely, the mean CMT and the mean initial superficial VD in the study by Toto et al were grossly different from those of the patient in the current study, ${ }^{10}$ while the initial visual acuity was worse in the study by Mastropasqua et al compared with our patient. ${ }^{17}$ The above differences indicate that the initial severity of microvasculopathy and the visual performance in retinal vascular diseases might influence the anti-angiogenesis effect of DEX.

The effectiveness of DEX in patients with DME has been illustrated in previous studies. ${ }^{5,7,8}$ For those patients with recalcitrant DME unresponsive to antiangiogenesis agent therapy, DEX still may slow the DME with morphological improvement. ${ }^{8}$ Although our patient was a new case and had not previously received anti-angiogenesis agent therapy, the edematous status progressed rapidly in three months thus DEX is theoretically more suitable than anti-angiogenesis agent therapy to retard the macular edema in our patient. In addition, the patient had previously received phacoemulsification; therefore, the steroid-induced cataract formation which can be induced by dexamethasone in the DEX was beyond our consideration. Also, the preoperative IOP was in the low-to-normal range so the chance to develop steroid-induced ocular hypertension after DEX implantation was unlikely in our patient. Since no contraindication was found and the edematous status was progressed, we selected DEX as the first-line treatment rather than anti-angiogenesis agent therapy. The decrease in vasculature with the improvement of both BCVA and CMT were observed for at least two months which might result from the anti-inflammatory effect of DEX in our opinion, and our finding may demonstrate the possibility that DEX can be the first treatment for DME in certain situations. 


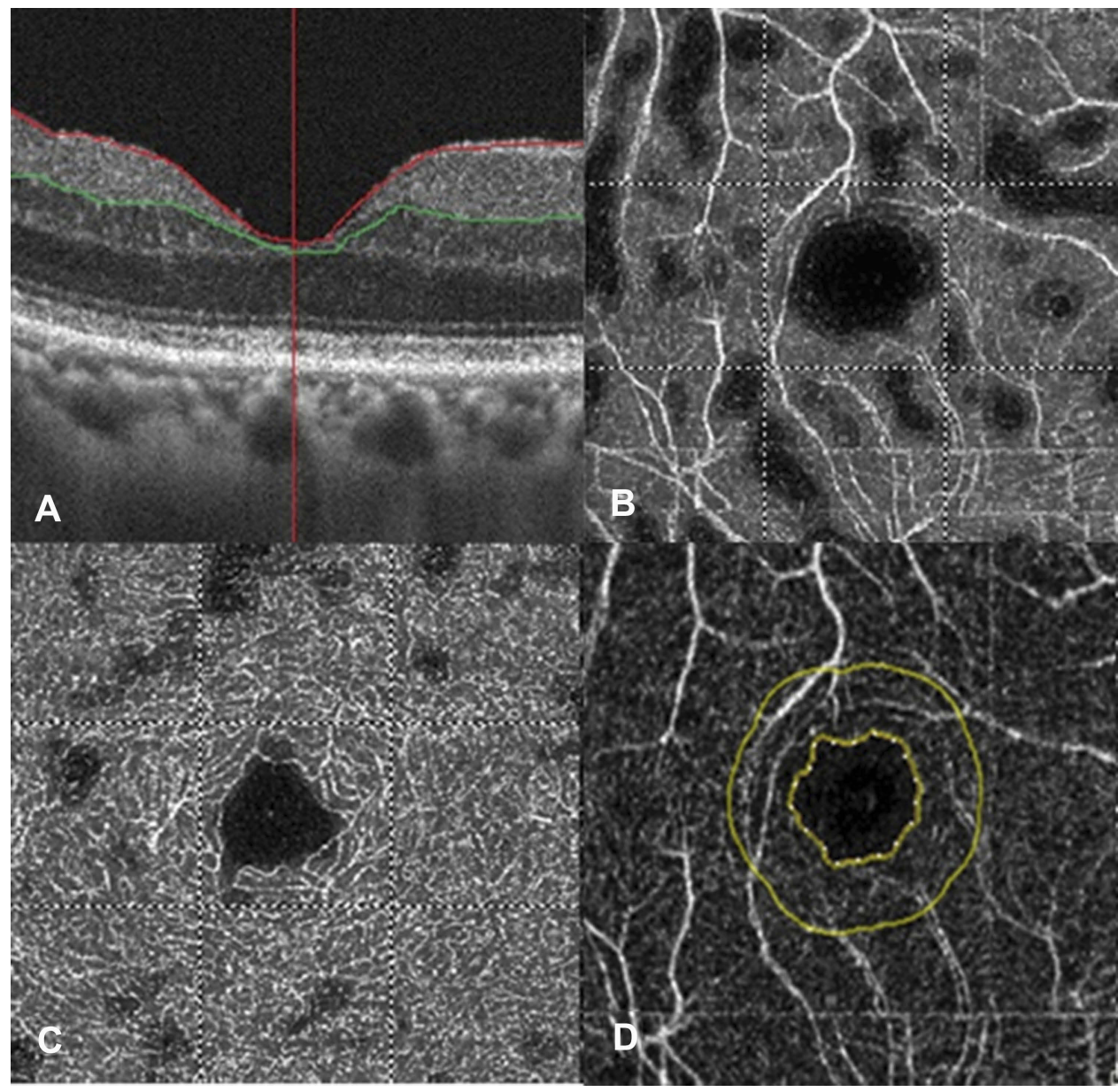

Figure 4 Macular conditions two months after dexamethasone implantation assessed using an optical coherence tomography angiography device. (A) Foveal thickness. (B) Superficial vessel density. (C) Deep vessel density. (D) Foveal avascular zone.

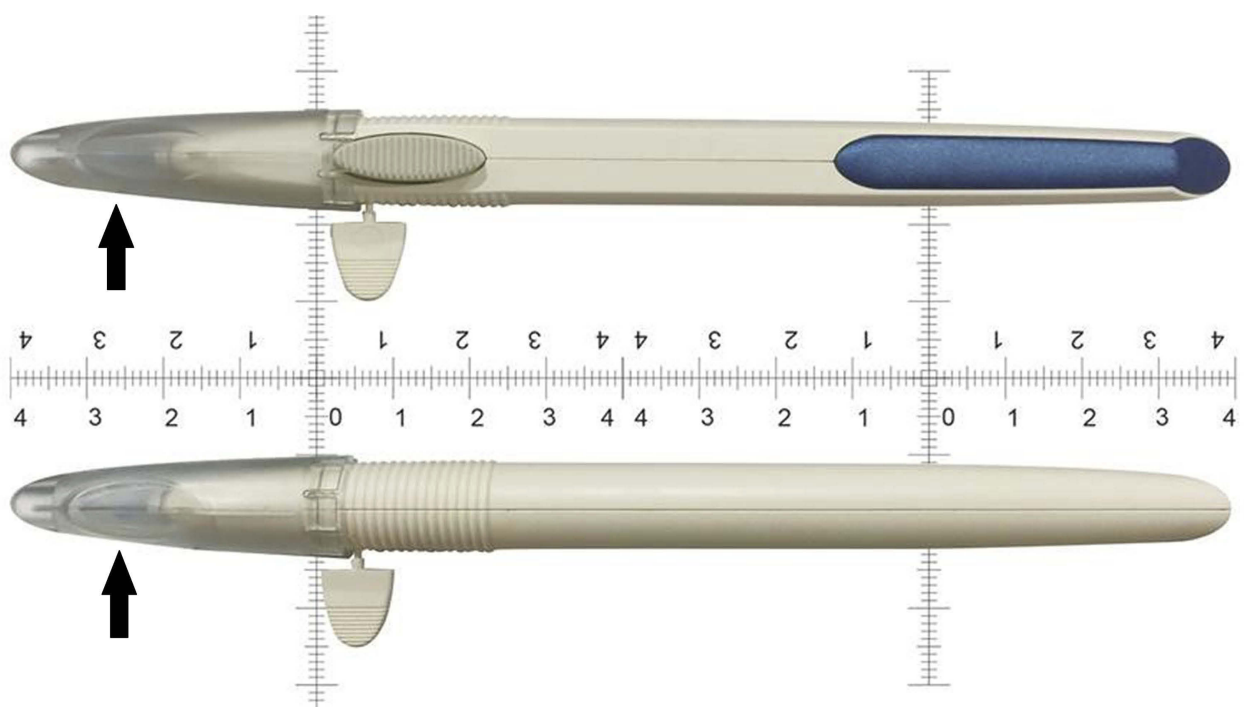

Figure 5 The appearance of dexamethasone intravitreal implant. Arrow: the dexamethasone-contain polymer device in the plastic cap. 


\section{Conclusion}

In conclusion, retinal VD decreased after the implantation of DEX in our patient with DME. Further large-scale studies are warranted to investigate the correlation between a decrease in VD and the recovery of visual parameters.

\section{Ethics approval and informed consent}

The study adhered to the declaration of Helsinki and was approved by the Institutional Review Board at the Show Chwan Memorial Hospital. In addition, written informed consent was obtained from the patient for publication of this case report including any accompanying images.

\section{Data availability}

Since the data included in the current report may contain some privacy information, the data will only be provided upon the request of editorial board.

\section{Abbreviations}

DEX, dexamethasone intravitreal implant; DME, diabetic macular edema; OCTA, optical coherence tomography angiography; BCVA, best-corrected visual acuity; CMT, central macular thickness; VD, vessel density.

\section{Author contributions}

All authors contributed to data analysis, drafting and revising the article, gave final approval of the version to be published, and agree to be accountable for all aspects of the work.

\section{Disclosure}

The authors report no conflicts of interest in this work.

\section{References}

1. Relhan N, Flynn HW Jr. The early treatment diabetic retinopathy study historical review and relevance to today's management of diabetic macular edema. Curr Opin Ophthalmol. 2017;28(3):205-212. doi:10.1097/ICU.0000000000000362

2. Whitcup SM, Cidlowski JA, Csaky KG, Ambati J. Pharmacology of corticosteroids for diabetic macular edema. Invest Ophthalmol Vis Sci. 2018;59(1):1-12. doi:10.1167/iovs.17-22259

3. Peres MB, Kato RT, Kniggendorf VF, et al. Comparison of optical coherence tomography angiography and fluorescein angiography for the identification of retinal vascular changes in eyes with diabetic macular edema. Ophthalmic Surg Lasers Imaging Retina. 2016;47 (11):1013-1019. doi:10.3928/23258160-20161031-05

4. Mane V, Dupas B, Gaudric A, et al. Correlation between cystoid spaces in chronic diabetic macular edema and capillary nonperfusion detected by optical coherence tomography angiography. Retina. 2016;36:S102-S110. doi:10.1097/IAE.0000000000001289
5. Mastropasqua R, Toto L, Borrelli E, et al. Morphology and function over a one-year follow up period after intravitreal dexamethasone implant (ozurdex) in patients with diabetic macular edema. PLoS One. 2015;10(12):e0145663. doi:10.1371/journal.pone.0145 663

6. Bucolo C, Gozzo L, Longo L, Mansueto S, Vitale DC, Drago F. Long-term efficacy and safety profile of multiple injections of intravitreal dexamethasone implant to manage diabetic macular edema: a systematic review of real-world studies. J Pharmacol Sci. 2018;138 (4):219-232. doi:10.1016/j.jphs.2018.11.001

7. Wickremasinghe SS, Fraser-Bell S, Alessandrello E, Mehta H, Gillies MC, Lim LL. Retinal vascular calibre changes after intravitreal bevacizumab or dexamethasone implant treatment for diabetic macular oedema. Br J Ophthalmol. 2017;101(10):1329-1333. doi:10.1136/ bjophthalmol-2016-309882

8. Iacono P, Parodi MB, Scaramuzzi M, Bandello F. Morphological and functional changes in recalcitrant diabetic macular oedema after intravitreal dexamethasone implant. Br J Ophthalmol. 2017;101 (6):791-795. doi:10.1136/bjophthalmol-2016-308726

9. Gill A, Cole ED, Novais EA, et al. Visualization of changes in the foveal avascular zone in both observed and treated diabetic macular edema using optical coherence tomography angiography. Int J Retina Vitreous. 2017;3:19. doi:10.1186/s40942-017-0074-y

10. Toto L, D'Aloisio R, Di Nicola M, et al. Qualitative and quantitative assessment of vascular changes in diabetic macular edema after dexamethasone implant using optical coherence tomography angiography. Int $J$ Mol Sci. 2017;18(6):1181. doi:10.3390/ ijms 18061181

11. Vangipuram G, Rezaei KA. Optical coherence tomography angiography as an imaging modality for evaluation of diabetic macular edema. J Ophthalmic Vis Res. 2017;12(4):359-360. doi:10.4103/ jovr.jovr_175_17

12. Lupidi M, Coscas F, Cagini C, Coscas G. Optical coherence tomography angiography in macular edema. Dev Ophthalmol. 2017;58:63-73. doi:10.1159/000455269

13. Chetrit M, Bonnin S, Mane V, et al. Acute pseudophakic cystoid macular edema imaged by optical coherence tomography angiography. Retina. 2017;38(10):2073-2080. doi:10.1097/IAE.00000 00000001829

14. Ghasemi Falavarjani K, Iafe NA, Hubschman JP, Tsui I, Sadda SR, Sarraf D. Optical coherence tomography angiography analysis of the foveal avascular zone and macular vessel density after anti-VEGF therapy in eyes with diabetic macular edema and retinal vein occlusion. Invest Ophthalmol Vis Sci. 2017;58(1):30-34. doi:10.1167/ iovs. 16-20579

15. Enders C, Lang GE, Dreyhaupt J, et al. Quantity and quality of image artifacts in optical coherence tomography angiography. PLoS One. 2019;14(1):e0210505. doi:10.1371/journal.pone.0210505

16. Fan Z, Sehm T, Rauh M, et al. Dexamethasone alleviates tumorassociated brain damage and angiogenesis. PLoS One. 2014;9(4): e93264. doi:10.1371/journal.pone.0093264

17. Mastropasqua R, Toto L, Di Antonio L, et al. Optical coherence tomography angiography microvascular findings in macular edema due to central and branch retinal vein occlusions. Sci Rep. 2017;7:40763. doi:10.1038/srep40763

18. Khadamy J, Abri Aghdam K, Falavarjani KG. An update on optical coherence tomography angiography in diabetic retinopathy. J Ophthalmic Vis Res. 2018;13(4):487-497. doi:10.4103/jovr. jovr_57_18

19. Balaratnasingam C, Inoue M, Ahn S, et al. Visual acuity is correlated with the area of the foveal avascular zone in diabetic retinopathy and retinal vein occlusion. Ophthalmology. 2016;123(11):2352-2367. doi:10.1016/j.ophtha.2016.07.008 


\section{Publish your work in this journal}

The International Medical Case Reports Journal is an international, peer-reviewed open-access journal publishing original case reports from all medical specialties. Previously unpublished medical posters are also accepted relating to any area of clinical or preclinica science. Submissions should not normally exceed 2,000 words or 4 published pages including figures, diagrams and references. The manuscript management system is completely online and includes a very quick and fair peer-review system, which is all easy to use. Visit http://www.dovepress.com/testimonials.php to read real quotes from published authors.

Submit your manuscript here: https://www.dovepress.com/international-medical-case-reports-journal-journal 